\title{
SERIAL PROSTATE SPECIFIC ANTIGEN MEASUREMENTS AND PROGRESSION IN UNTREATED CONFINED (STAGES T0 TO 3NxM0, GRADES 1 TO 3) CARCINOMA OF THE PROSTATE
}

\author{
CHR. H. BANGMA, W. C. J. HOP AND F. H. SCHRODER \\ From the Departments of Urology and Epidemiology and Biostatistics, Erasmus University and Academic Hospital, Rotterdam, \\ The Netherlands
}

ABSTRACT

Purpose: The contribution of serial prostate specific antigen (PSA) determinations was studied to obtain better understanding of the natural history of clinically confined prostate carcinoma.

Materials and Methods: Serial PSA determinations in 29 patients with untreated confined prostate carcinoma were correlated to the clinical course for a mean of 39 months.

Results: Disease progressed locally in 13 patients after a mean of 31 months. Metastatic progression was not observed. Neither grade, stage, PSA changes nor initial PSA showed significant differences with respect to interval of progression between patients with and without progression.

Conclusions: PSA does not parallel clinical progression in patients selected for watchful waiting.

KEY WORDS: prostatic neoplasms; antigens, neoplasm; follow-up studies; neoplasm metastasis

Our study was done to contribute to a better understanding of the natural history of clinically confined prostate carcinoma and to determine the contribution of serial prostate specific antigen (PSA) measurements. For this reason PSA determinations were correlated with the clinical course in 29 patients in whom a policy of watchful waiting was applied. In several studies increasing PSA levels predicted later clinical progression. ${ }^{1-4}$ The interval between both events depends on tumor stage at diagnosis or treatment. Regarding the correlation of PSA increase and progression in stages T1 and T2 tumors with time, little information is available in the literature. With regard to the uncertainties of identifying local progression it may well be possible that an increase in PSA level is a more accurate indicator of progressive disease in these patients.

\section{PATIENTS AND METHODS}

We evaluated retrospectively 29 patients with histologically confirmed adenocarcinoma of the prostate from the time of diagnosis until the time of first treatment, if any, for cancer-related progression. Mean patient age was 74 years (range 58 to 85), and mean followup was 39 months (range 11 to 73). Stage was assessed clinically by digital rectal examination according to the 1992 TNM classification. ${ }^{5}$ In $15 \mathrm{pa}-$ tients with stage T1a disease previously unsuspected carcinoma was found in the transurethral resection specimens. Grade was determined according to the Anderson classification. ${ }^{6}$ Metastatic disease was excluded by a normal chest $x$-ray and a normal bone scan. The decision not to treat at diagnosis was made by the urologist in discussion with the patient and his family, with respect to patient age, general health, clinical stage and patient preference. All patients were considered to have a probable survival of at least 1 year.

Followup. Patients usually were followed clinically twice yearly (mean 2.7 annual visits, range 1.4 to 4.3 ). Physical examination was done, including digital rectal examination, and serum PSA and alkaline phosphatase levels were assessed at each visit from the time of diagnosis. When no PSA value was available at diagnosis (baseline value) but PSA was obtained 1 or more months afterwards, this value was extrapolated by

Accepted for publication March 24, 1995. linear regression from the logarithmically transformed PSA values versus time during the first 2 years after diagnosis for individual patients. This method was evaluated by applying the procedure to 10 patients in whom a baseline value of PSA was available. In this group the correlation between the extrapolated value obtained and the actual value was good $(r=0.98)$, indicating its validity. Thus, the values obtained when no baseline value was available were used in all further calculations when a baseline value was needed (except for 1 patient in whom PSA followup began 2 years after diagnosis). Bone scan and chest $x$-ray were repeated regularly and when clinically indicated. In 14 patients serial transrectal ultrasonic volumetry of the prostate was performed.

Staging and progression. Of 29 patients whose disease was classified according to the 1992 TNM system 15 had stage T1a, 13 stage T2 and 1 stage T3 tumor. There were 11 grade 1,15 grade 2 and 1 grade 3 cancers (grade was unknown in 2 patients). Mean patient age was 74 years (range 58 to 85 ). The distribution of age and grade with the $T$ categories is summarized in the table.

Local and metastatic progression was evaluated. Subjective progression, like obstructive micturition or pain, was considered for treatment decisions. Local subjective progression occurred in most patients at the same time as or later than local objective progression. Local objective progression was defined as an increase in $\mathrm{T}$ category, when prostate size increased on digital rectal examination by $25 \%$ or more of the products of the 2 largest perpendicular parameters, or by an ultrasound measured volume of more than $40 \%$. Objective metastatic progression was diagnosed by the appearance of new lesions on a bone scan. Bone scans were performed for initial staging and afterwards when indicated only. For the purpose of defining clinical progression, an increase in serum

Distribution of patient age and grade with $T$ categories

\begin{tabular}{lccccc}
\hline $\begin{array}{c}\text { T } \\
\text { Category }\end{array}$ & No. Pts. & $\begin{array}{c}\text { Mean Age } \\
\text { (yrs.) }\end{array}$ & \multicolumn{3}{c}{ Grade (No. pts.) } \\
\hline T1a & 15 & 74 & 7 & 2 & 3 \\
T2 & 11 & 77 & 3 & 6 & - \\
T3 & 3 & 72 & 1 & 2 & - \\
\hline
\end{tabular}


markers alone was not considered relevant. In case of progression the patients remained in the study up to the time of initial therapy, which may have been started because of subjective or objective local or distant progression.

During the course of this study only 1 patient died of a cause not related to disease (another malignancy). The dayto-day coefficient of variation of the Hybritech PSA assay related to 3 "stable" standards for 50 days varied between 6.9 and $18.8 \%$.

Statistical analysis included Kaplan-Meier curves for analysis of interval to progression in patients according to stage and grade. The changes in PSA and prostate volume were expressed by the slopes of the log-linear curves versus time. In 1 patient 1 serial PSA value was extremely low without evident reason. This outlier was not included in the calculations of PSA slopes. The Mann-Whitney test was used to evaluate differences among various groups of patients regarding these slopes. The level of statistical significance was set at $p=0.05$ (2-tailed). The relationship between progression rates and PSA level or its change from the baseline value was evaluated using Cox regression allowing for time-dependent variables. ${ }^{7}$ The relationship between PSA levels and simultaneous prostate volume measurements, allowing for inter-patient and intra-patient differences, was assessed by computer using regression analysis.

\section{RESULTS}

During followup 13 of 29 patients had local progression after a mean of $\mathbf{3 1}$ months. Metastatic progression was not noted. Of these patients with progression 6 started therapy: 5 for subjective symptoms at or within 3 months after progression and 1 for objective progression only. Two patients with local objective and subjective progression did not begin therapy. Disease did not progress in 16 patients (mean followup 40 months).

Figure 1 shows the Kaplan-Meier curves for interval to progression for all patients, and for stage $\mathrm{T} 1$ versus stages $\mathrm{T} 2$ to T3 and grade 1 versus grades 2 to 3 cancer. There was no statistical difference in interval to progression among the different grades or the different $T$ categories. The mean number of PSA recordings per patient was 8.7. Figure 2 shows the scatterplots of PSA with time in linear plotting for patients with and without clinical progression. Slopes of PSA regression curves comparing progressive and nonprogressive disease were not significantly different. Figure 3 shows a plot of the PSA doubling time for the individual patients, separated for those with and without progression. Also, a percentage PSA increase from a baseline of $20 \%$ with time was tested as an indicator of progression (fig. 4). At no point on the individual curves was it possible to predict clinical progression. The same applied to the cutoff level of a $50 \%$ increase in PSA with time.

Longitudinal measurements of ultrasonic prostatic volume were made in 14 patients. The mean annual increase in prostatic volume in these patients was $15 \%$. The mean increase in volume was not significantly different between patients with and without progression. A volume increase of more than $40 \%$, which would qualify a patient for clinical progression on the basis of transrectal ultrasonography, was not observed. There was a weak, although significant, correlation between volume and PSA (fig. 5). Changes in both parameters within patients, however, were not significantly related to each other.

\section{DISCUSSION}

In our study an attempt was made to identify the rate and interval of progression in 29 patients with locally confined prostate cancer treated by a policy of watchful waiting. In no instance was systemic progression observed during an average observation of 31 months. Local progression was identifiable as an increase in $T$ category or as a palpable increase in prostate size in conjunction with subjective progression of the tumor. ${ }^{8}$ A volume increase on transrectal ultrasound of $40 \%$ or more was not observed. ${ }^{9}$ PSA increase was independently studied and compared with clinical progression.

Our information presented is likely to be biased by patient selection and is certainly not representative of men for whom radical prostatectomy would normally be recommended. However, the data may be compatible with patient populations presented in other surveillance studies, such as those summarized in the overview by Chodak et al. ${ }^{10}$ The selection bias is reflected in the high average patient age, large proportion of grade 1 and stage Tla tumors, and absence of high grade lesions. The median interval to progression for stages $\mathrm{T} 1$ and $\mathrm{T} 2$ to $\mathrm{T} 3$ cancer patients was approximately 42 months for both groups (fig. 1), which is shorter than that reported by Lowe and Listrom, ${ }^{11}$ and Schröder in 1993 for stage T1a tumors (approximately 13 years), ${ }^{12}$ and by Whitmore et $\mathrm{al}^{13}$ for stage $\mathrm{T} 2$ tumors (approximately 10 years).

Several methods of analyzing PSA increase have been applied. PSA velocity, which represents the absolute annual PSA increase, was used by Carter et al in a select group of 52 men to discriminate between prostate carcinoma and benign disease. ${ }^{14} \mathrm{~A}$ discriminatory cutoff value of $0.75 \mathrm{ng} . / \mathrm{ml}$. per year was not confirmed by Brawer et al in a communitybased population of 701 men. ${ }^{15}$ In that study the participants underwent further evaluation by digital rectal examination when the PSA level increased more than $20 \%$ within 1 year. Prostate carcinoma was found eventually in $5 \%$ of these men. Oesterling et al noted an age independent annual increase in PSA among several age groups in a community-based cohort of 376 men. ${ }^{16}$ The mean annual increase in PSA was 5.1 to $11.4 \%$ but it was extremely variable partly due to the fact that the followup was 1 year and only 2 PSA measurements were obtained. In $1.1 \%$ of the cases carcinoma developed, with a mean annual PSA increase of $59 \%$.

In our study increases in PSA with time could not discriminate between nonprogressive, often incidentally found, lo-
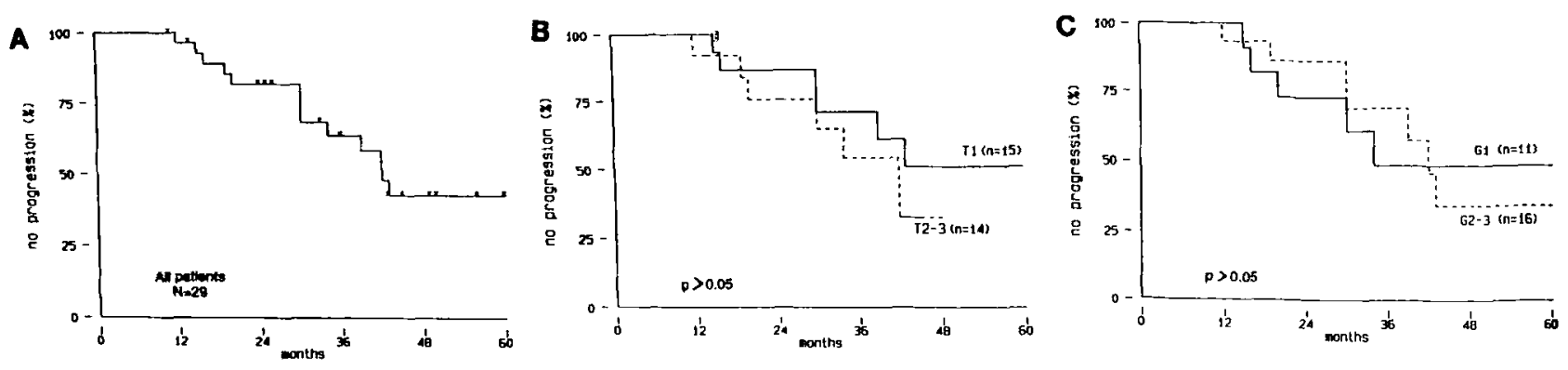

FIG. 1. Kaplan-Meier curves for interval to progression for all patients (A), separated for stage T1 versus stages $T 2$ to $3(B)$ and for grade 1 versus grades 2 to $3(C)$. Tick marks along curves denote ends of followup of some patients. 
A

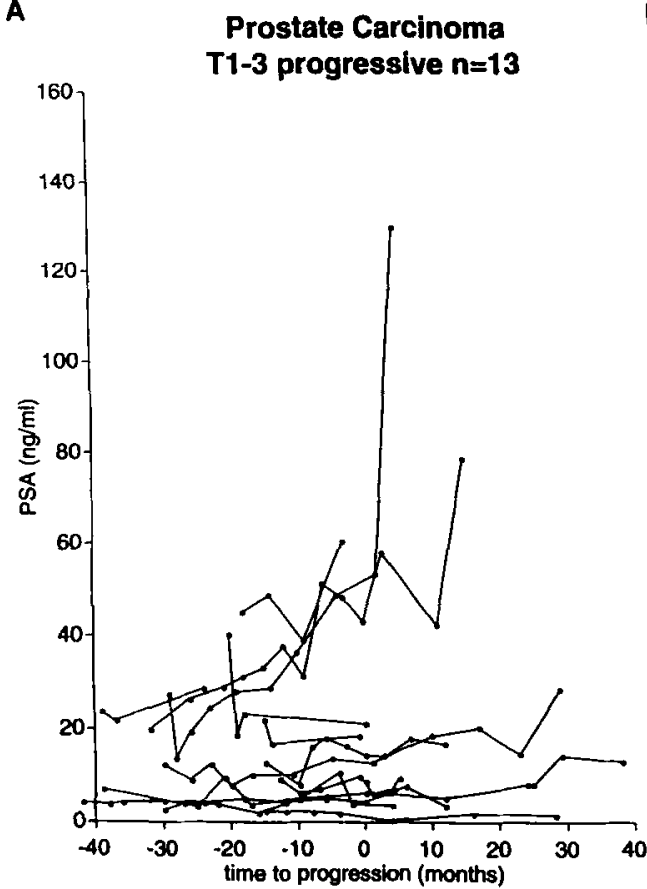

B
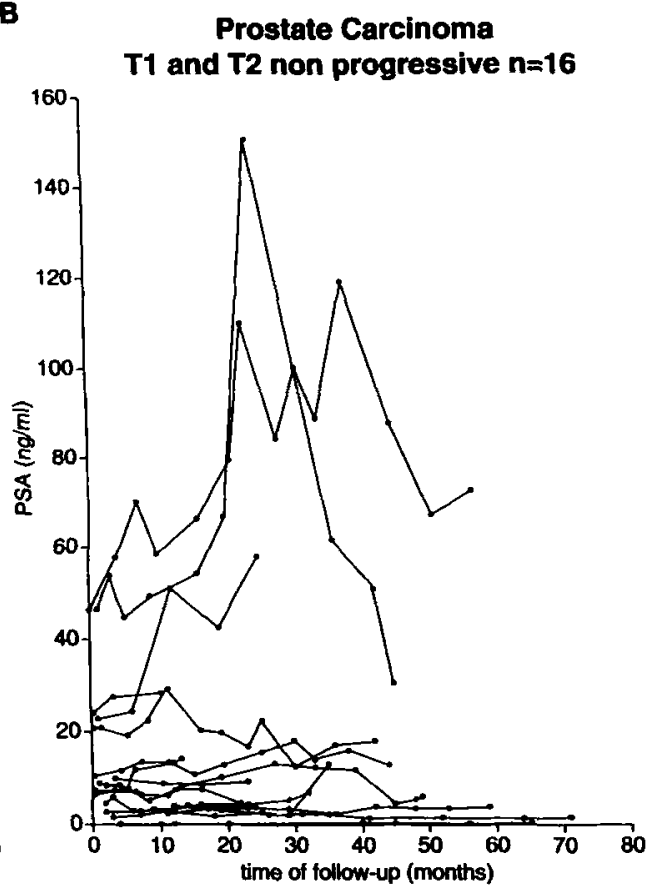

F1G. 2. Scatterplots of PSA with time in linear plotting for patients with $(A)$ and without $(B)$ clinical progression

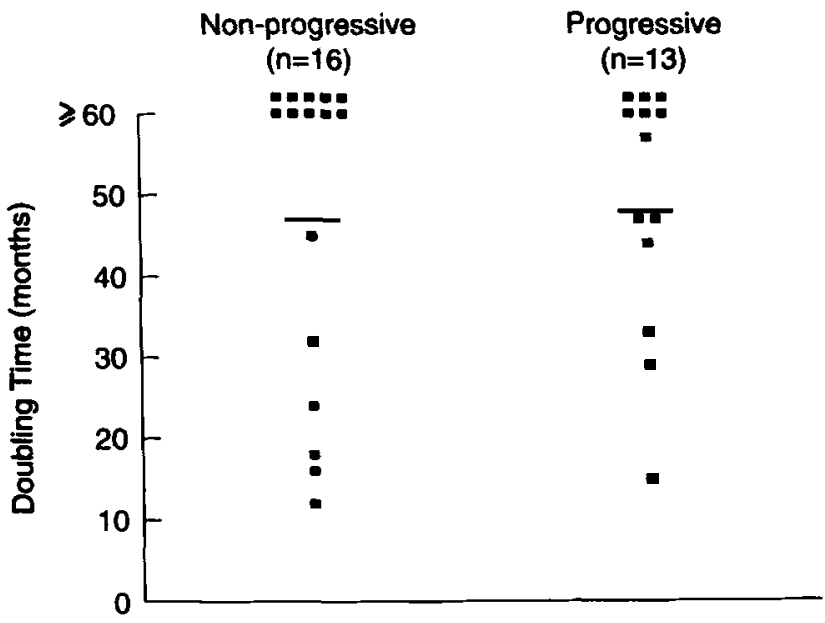

Fig. 3. Plot of PSA doubling times for patients with and without progression.

calized prostate carcinoma and progressive disease. Also, the annual PSA increases during year 1, 2 or 3 of the evaluation were not markers for progression, which means that although there was a wide variety in PSA velocity and PSA doubling times (fig. 3), these parameters were not useful in these cases for this study period.

Our report confirms the PSA doubling time for organconfined prostate carcinoma found in other studies. In 43 untreated patients with prostate carcinoma Schmid et al observed that 20 of 28 with organ-confined carcinoma had a PSA doubling time of more than 4 years. ${ }^{17}$ The PSA doubling time in cases of higher grades or stages was faster than for others. Although 14\% of all patients had a stable PSA level it was concluded that prostate carcinoma has an exponential growth rate. In our study 17 of 29 patients had a PSA doubling time of more than 4 years. In the study of Cadeddu et

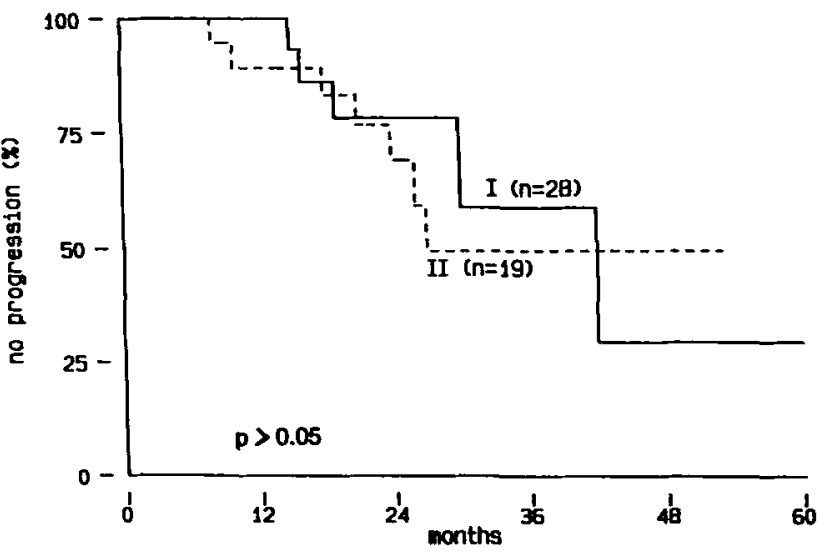

F1G. 4. Kaplan-Meier curves for interval to progression for 19 patients from time of PSA increase of $20 \%$ or more without prior clinical progression $(I I)$ versus 28 patients during period as long as PSA increase was less than $20 \%(f)$

al the PSA doubling time in 11 patients with confined disease versus 5 with progression to metastasis was not statistically different. ${ }^{18}$ Carter et al mentioned that the linear increase in PSA level between patients with benign prostatic hyperplasia and prostatic carcinoma (up to diagnosis) was statistically different but from the PSA curves it is obvious that this PSA velocity has no prognostic value. ${ }^{14}$

A low PSA level has been associated with long-term survival in a study by Belville et al of 26 patients with stage $\mathrm{A1}$ prostate carcinoma. ${ }^{19}$ The patients had an age distribution similar to that of our study population (median age 72 years). All tumors had well differentiated histology. Of the PSA levels $96 \%$ were less than $3.0 \mathrm{ng} / \mathrm{ml}$. Evaluation after a median followup of 12.5 years showed that in all patients the prostate carcinoma remained localized, although $6(23 \%)$ had progression to a palpable stage. Of our 14 patients with PSA 


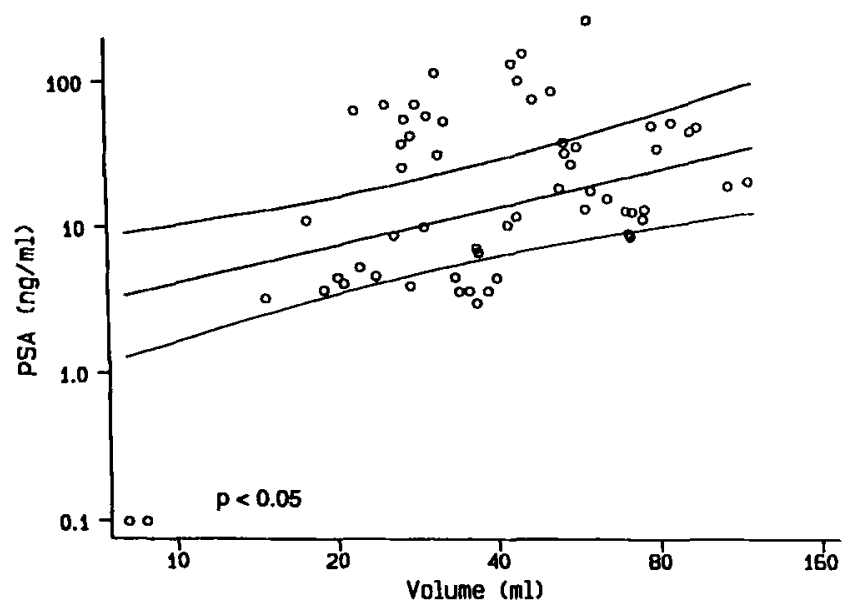

Fig. 5. Mean PSA level according to volume. Curved lines denote 95\% confidence interval for mean PSA. Disregarding outlying 2 observations of 1 patient (lower left corner), relationship was still significant $(\mathrm{p}<0.05)$.

values less than $10 \mathrm{ng} . / \mathrm{ml} .5$ had progression. Only physician dependent initiation of therapy was found to be more frequent in patients with higher PSA values. Transrectal ultrasonic volumetry was not helpful as a parameter for progression.

\section{CONCLUSIONS}

In our study population, and probably in similarly select groups of patients, watchful waiting is considered justified. The absence of metastatic progression is at variance with other findings in the literature. ${ }^{20,21}$ On the basis of our study however, it appears to be safe to follow patients with PSA determinations, rectal examination, and the intermittent evaluation of related symptoms and signs. It must be realized that clinical progression may occur without changes in serum PSA.

\section{REFERENCES}

1. Stamey, T. A., Graves, H. C. B., Wehner, N., Ferrari, M. and Freiha, F. S.: Early detection of residual prostate cancer after radical prostatectomy by an ultrasensitive assay for prostate specific antigen. J. Urol., 149: 787, 1993.

2. Brawer, M. K. and Lange, P. H.: Prostate-specific antigen in management of prostatic carcinoma. Urology, suppl., 33: 11 1989.

3. Partin, A. W., Pound, C. R., Clemens, J. Q., Epstein, J. I. and Walsh, P. C.: Serum PSA after anatomic radical prostatectomy. The Johns Hopkins experience after 10 years. Urol. Clin. N. Amer., 20: 713, 1993.

4. Davidson, P. J. T., Vis, J., Fossa, S., Kurth, K. H. and Schröder, F. H.: Progression in untreated D1 (T0-4 N1-3 M0) carcinoma of the prostate. Lancet, 1994, submitted for publication.
5. Schröder, F. H., Hermanek, P., Denis, L., Fair, W. R., Gospodarowicz, M. K and Pavone-Macaluso, M.: The TNM classification of prostate carcinoma. Prostate, suppl., 4: 129, 1992.

6. Brawn, P. N., Ayala, A. G., von Eschenbach, A. H., Hussey, D. H. and Johnson, D. E.: Histologic grading study of prostate carcinoma: the development of a new system and comparison with other methods-a preliminary study. Cancer, 49: 525, 1982.

7. Cox, D. R.: Regression models and life-tables. J. Roy. Stat. Soc., 34: 187, 1972.

8. Newling, D. W. W.: Criteria of response to treatment in advanced prostatic cancer. Bailliere's Clin. Oncol., 2: 505, 1988.

9. Carpentier, P. J. and Schröder, F. H.: Transrectal ultrasonography in the followup of prostatic carcinoma patients: a new prognostic parameter? J. Urol., 131: 903, 1983.

10. Chodak, G. W., Thisted, R. A., Gerber, G. S., Johansson, J. E., Adolfsson, J., Jones, G. W., Chisholm, G. D., Moskovitz, B., Livne, P. M. and Warner, J.: Results of conservative management of clinically localized prostate cancer. New Engl. J. Med., 330: 242, 1994.

11. Lowe, B. A. and Listrom, M. B.: Incidental carcinoma of the prostate: an analysis of the predictors of progression. J. Urol., 140: $1340,1988$.

12. Schröder, F. H.: The natural history of incidental prostatic carcinoma. In: Incidental Carcinoma of the Prostate. Edited by $J$. E. Altwein, P. Faul and W. Schneider. New York: SpringerVerlag, pp. 56-62, 1991.

13. Whitmore, W. F., Jr., Warner, J. A. and Thompson, I. M., Jr. Expectant management of localized prostatic cancer. Cancer, 67: 1091, 1991.

14. Carter, H. B., Pearson, J. C., Metter, E. J., Brant, L. J., Chan D. W., Andres, R., Fozard, J. L. and Walsh, P. C.: Longitudinal evaluation of prostate specific antigen levels in men with and without prostate disease. J.A.M.A., 267: 2215, 1992.

15. Brawer, M. K. Beatie, J., Wener, M. H., Vessella, R. J., Preston, S. D. and Lange, P. H.: Screening for prostate carcinoma with prostate specific antigen: result of the second year. J. Urol., 150: 106, 1993.

16. Oesterling, J. E., Chute, C. G., Jacobsen, S. J., Guess, H. A., Panser, L. A., Johnson, C. L. and Lieber, M. M.: Longitudinal changes in serum PSA (PSA velocity) in a community-based cohort of men. J. Urol, part 2, 149: 412A, abstract 799, 1993.

17. Schmid, H.-P., McNeal, J. E. and Stamey, T. A.: Observations on the doubling time of prostate cancer as measured by serial prostate-specific antigen in untreated patients with prostate cancer. J. Urol., part 2, 147: 387A, abstract 699, 1993.

18. Cadeddu, J. A., Pearson, J. D., Partin, A. W., Epstein, J. I. and Carter, H. B.: Relationship between changes in prostatespecific antigen and prognosis of prostatic cancer. Urology, 42: 383, 1993.

19. Belville, W. D., Vaccaro, J. A. and Kiesling, V. J., Jr.: Prostatespecific antigen and digital rectal examination in long-term follow-up of stage A1 prostatic carcinoma. Urology, 39: 586, 1992.

20. Blute, M. L., Zincke, H. and Farrow, G. M.: Long-term followup of young patients with stage A adenocarcinoma of the prostate. J. Urol., 136: 840, 1986.

21. Epstein, J. I., Paull, G., Eggleston, J. C. and Walsh, P. C.: Prognosis of untreated stage A1 prostatic carcinoma: a study of 94 cases with extended followup. J. Urol., 136: 837, 1986. 